\title{
High energy solutions for the fourth-order elliptic equations in $R^{N}$
}

Bitao Cheng*

\section{"Correspondence:}

chengbitao2006@126.com

Department of Mathematics and

Information Science, Qujing Normal

University, Qujing, Yunnan 655011,

P.R. China

\begin{abstract}
In the present paper, we study the following fourth-order elliptic equations: $\Delta^{2} u-\Delta u+V(x) u=f(x, u)$, for $x \in R^{N}, u(x) \in H^{2}\left(R^{N}\right)$, where $V \in C\left(R^{N}, R\right), f \in C\left(R^{N} \times R, R\right)$. Under more relaxed assumptions on $f(x, u)$, by using some special techniques, a new existence result of high energy solutions is obtained via the symmetric mountain pass theorem.
\end{abstract}

Keywords: fourth-order elliptic equations; symmetric mountain pass theorem; high energy solutions

\section{Introduction and main results}

In this paper, we consider the following fourth-order elliptic equations:

$$
\left\{\begin{array}{l}
\Delta^{2} u-\Delta u+V(x) u=f(x, u), \quad \text { for } x \in R^{N} \\
u(x) \in H^{2}\left(R^{N}\right)
\end{array}\right.
$$

where $\Delta^{2}:=\Delta(\Delta)$ is the biharmonic operator, $V \in C\left(R^{N}, R\right), f \in C\left(R^{N} \times R, R\right)$ satisfy some further conditions.

When $\Omega$ is a smooth bounded domain in $R^{N}$, the problem

$$
\begin{cases}\Delta^{2} u+c \Delta u=f(x, u), & \text { in } \Omega, \\ u=\Delta u=0, & \text { on } \partial \Omega,\end{cases}
$$

arises in many applications from mathematical physics, which is usually used to describe some phenomena appearing in various physical, engineering, and other sciences. In [1, 2], Lazer and Mckenna firstly proposed and studied the problem (1.2) of periodic oscillations and traveling waves in a suspension bridge. It was pointed out in [3-6] that the problem (1.2) furnishes a good model to the study of the static deflection of an elastic plate in a fluid. Moreover, Ahmed and Harbi presented that the problem (1.2) can also be applied to engineering, such as communication satellites, space shuttles and space stations equipped with large antennas mounted on long flexible beams in [7]. Further, there is a large quantity of studies on the existence and multiplicity of solutions in the literature, we refer the readers to $[8-21]$ and the references therein.

\section{空 Springer}

(C) 2014 Cheng; licensee Springer. This is an Open Access article distributed under the terms of the Creative Commons Attribution License (http://creativecommons.org/licenses/by/4.0), which permits unrestricted use, distribution, and reproduction in any medium, provided the original work is properly credited. 
Recently, Lü [22] considered a class of biharmonic elliptic systems with Sobolev critical exponent on a bounded domain

$$
\begin{cases}\Delta^{2} u=F_{u}(u, v)+\lambda|u|^{q-2} u, & x \text { in } \Omega ; \\ \Delta^{2} v=F_{v}(u, v)+\delta|v|^{q-2} v, & x \text { in } \Omega ; \\ u=\frac{\partial u}{\partial n}, \quad v=\frac{\partial v}{\partial n}, & x \text { on } \partial \Omega ;\end{cases}
$$

where $\Omega \subset R^{N}(N \geq 5)$ is a bounded domain with smooth boundary $\partial \Omega, 0 \in \Omega, F \in$ $C^{1}\left(R^{2}, R^{+}\right)$is homogeneous of degree $2_{*}\left(2_{*}=\frac{2 N}{N-4}\right.$ denotes the Sobolev critical exponent). The existence and multiplicity results of nontrivial solutions for the system (1.3) were obtained by using the variational methods and the Nehari manifold.

The above mentioned interesting studies for the fourth-order elliptic equations are based on boundness of $\Omega \subset R^{N}$. Some authors had studied the fourth-order elliptic equations in the whole space $R^{N}$. In [23], Chabrowski and Marcos do Ó considered the following fourth-order elliptic problems in $R^{N}$ :

$$
\left\{\begin{array}{l}
\Delta^{2} u-\lambda g(x) u=f(x)|u|^{p-2} u, \quad \text { in } R^{N} \\
u \in D^{2,2}\left(R^{N}\right)-\{0\}
\end{array}\right.
$$

where $\lambda>0$ and $p=\frac{2 N}{N-4}$ the Sobolev critical exponent. By employing the mountain pass theorem and the constrained minimization together with the concentration-compactness principle, the existence of two solutions were obtained. In [24], Pimenta and Soares considered the following superlinear fourth-order elliptic equations:

$$
\left\{\begin{array}{l}
\epsilon^{4} \Delta^{2} u+V(x) u=f(u), \quad \text { in } R^{N} \\
u \in H^{2}\left(R^{N}\right),
\end{array}\right.
$$

where $\epsilon>0, N \geq 5$, and $f$, and $V$ satisfy some certain assumptions. Under a weak version of the Ambrosetti-Rabinowitz condition, by means of variational methods, a family of solutions was proved to exist and to concentrate at a point in the limit. Very recently, some authors studied the following fourth-order elliptic equations in $R^{N}$ :

$$
\left\{\begin{array}{l}
\Delta^{2} u-\Delta u+\lambda V(x) u=f(x, u), \quad \text { in } R^{N} \\
u \in H^{2}\left(R^{N}\right)
\end{array}\right.
$$

where $\lambda \geq 1$ is a parameter and $f \in C\left(R^{N}, R\right)$. For the potential $V(x)$, they usually assumed one of the following assumptions.

$\left(\mathrm{V}_{1}\right) \quad V \in C\left(R^{N}, R\right)$ satisfies $\inf V(x) \geq V_{0}>0$ and for each $M>0$, meas $\left\{x \in R^{N}: V(x) \leq\right.$ $M\}<+\infty$, where $V_{0}$ is a constant and meas denote the Lebesgue measure in $R^{N}$.

$\left(\mathrm{V}_{1}^{\prime}\right) V \in C\left(R^{N}, R\right)$ satisfies inf $V(x) \geq V_{0}>0$ and there exists a constant $b>0$ such that the set $\left\{x \in R^{N}: V(x) \leq b\right\}$ is nonempty and meas $\left\{x \in R^{N}: V(x) \leq b\right\}<+\infty$, where $V_{0}$ is a constant and meas denote the Lebesgue measure in $R^{N}$.

Yin and $\mathrm{Wu}$ [25] obtained three new results of the existence of infinitely many high energy solutions for the equation (1.6) with $\lambda=1$ and $V$ satisfying $\left(\mathrm{V}_{1}\right)$. Soon after, Ye and Tang [26] unified and improved their results by means of fountain theorem. To be precise, they assumed the following assumptions. 
$\left(\mathrm{f}_{1}\right)$ There exist $C_{1}>0$ and $p \in\left(2,2_{*}\right)$ such that

$$
|f(x, t)| \leq C_{1}\left(|t|+|t|^{p-1}\right), \quad \forall(x, t) \in R^{N} \times R
$$

where $2_{*}=\frac{2 N}{N-4}$, if $N>4 ; 2_{*}=+\infty$, if $N \leq 4$.

$\left(\mathrm{f}_{2}^{\prime}\right)$ There exists $L_{0}>0$ such that $\inf _{x \in R^{N},|t|=L_{0}} F(x, t)>0$, where $F(x, t)=\int_{0}^{t} f(x, s) d s$.

$\left(f_{3}^{\prime}\right)$ There exists $\mu_{0}>2$ such that

$$
\mu_{0} F(x, t) \leq f(x, t) t, \quad \text { for a.e. } x \in R^{N} \text { and } \forall|t| \geq L_{0}
$$

$\left(\mathrm{f}_{4}\right) f(x,-t)=-f(x, t)$ for all $(x, t) \in R^{N} \times R$.

We restate the corresponding result in [26] as follows.

Theorem A (see [26, Theorem 1.1]) Assume that $\left(\mathrm{V}_{1}\right),\left(\mathrm{f}_{1}\right),\left(\mathrm{f}_{2}^{\prime}\right)-\left(\mathrm{f}_{3}^{\prime}\right)$, and $\left(\mathrm{f}_{4}\right)$ hold. Then the problem (1.1) possesses infinitely many solutions $\left\{u_{k}\right\}$ such that

$$
\frac{1}{2} \int_{R^{N}}\left(\left|\Delta u_{k}\right|^{2}+\left|\nabla u_{k}\right|^{2}+V(x) u_{k}^{2}\right) d x-\int_{R^{N}} F\left(x, u_{k}\right) d x \rightarrow+\infty, \quad \text { as } k \rightarrow \infty .
$$

More recently, Liu et al. in [27] studied the existence and multiplicity of nontrivial solutions of (1.6) for large $\lambda$ and $V$ satisfying $\left(\mathrm{V}_{1}^{\prime}\right)$. These results had been subsequently unified and improved by Ye and Tang in [28]. For the sublinear case of the fourth-order elliptic equation (1.1), by using the genus properties in critical point theory, infinitely many small negative energy solutions were also established; we refer the readers to $[26,28,29]$.

Remark 1 The condition $\left(\mathrm{V}_{1}\right)$, which contains the coercivity condition: $V(x) \rightarrow \infty$ as $|x| \rightarrow \infty$, was first introduced by Bartsch and Wang in [30] to ensure the compactness of embedding of the working space. Furthermore, when replacing $\left(\mathrm{V}_{1}\right)$ by the weaker condition $\left(V_{1}^{\prime}\right)$, this situation becomes more delicate due to the lack of compactness. Although the compactness of embedding of the working space fails, thanks to the fact that the parameter $\lambda$ can be taken sufficiently large, two main theorems as regards the existence and multiplicity of nontrivial solutions of (1.6) were obtained via variational methods successfully.

Motivated by the spirit of [25-28], we shall consider the fourth-order elliptic equations with Bartsch type potential (that is, $V$ satisfies the condition $\left(\mathrm{V}_{1}\right)$ ) and superlinear nonlinearity case in the whole space $R^{N}$, and we are interested in high energy solutions of the problem (1.1). Under more relaxed assumptions on the nonlinear $\operatorname{term} f$, we will present a new proof technique to construct high energy solutions for the problem (1.1).

Next, we give some notations. Define the function space

$$
H^{2}\left(R^{N}\right):=\left\{u \in L^{2}\left(R^{N}\right):|\nabla u|, \Delta u \in L^{2}\left(R^{N}\right)\right\}
$$

with the standard inner product and the norm

$$
\langle u, v\rangle_{H^{2}}=\int_{R^{N}}(\Delta u \Delta v+\nabla u \nabla v+u v) d x, \quad\|u\|_{H^{2}}=\langle u, u\rangle_{H^{2}}^{\frac{1}{2}}
$$


whenever $u, v \in H^{2}\left(R^{N}\right)$. Let

$$
E:=\left\{u \in L^{2}\left(R^{N}\right): \int_{R^{N}}\left((\Delta u)^{2}+|\nabla u|^{2}+V(x) u^{2}\right) d x<+\infty\right\} .
$$

Then $E$ is a Hilbert space with the inner product and the norm

$$
\langle u, v\rangle_{E}=\int_{R^{N}}(\Delta u \Delta v+\nabla u \nabla v+V(x) u) d x, \quad\|u\|_{E}=\langle u, u\rangle_{E}^{\frac{1}{2}} .
$$

Obviously, the embedding

$$
E \hookrightarrow L^{s}\left(R^{N}\right), \quad 2 \leq s \leq 2 *
$$

is continuous. Hence, for any $s \in\left[2,2_{*}\right]$, there is a constant $a_{s}>0$ such that

$$
\|u\|_{L^{s}} \leq a_{s}\|u\|_{E}
$$

It is well known that a weak solution for the problem (1.1) is a critical point of the following functional $I$ defined on $E$ :

$$
I(u)=\frac{1}{2} \int_{R^{N}}\left(|\Delta u|^{2}+|\nabla u|^{2}+V(x) u^{2}\right) d x-\int_{R^{N}} F(x, u) d x, \quad \forall u \in E .
$$

We say that a weak solutions sequence $\left\{u_{n}\right\} \subset E$ for the problem (1.1) is a sequence of high energy solutions if the energy $I\left(u_{n}\right) \rightarrow+\infty$ as $n \rightarrow \infty$.

In order to reduce the statements of our result, we make the following assumptions.

$\left(f_{2}\right)$ There exists $2<\theta<2$ such that $\liminf _{|t| \rightarrow+\infty} \frac{F(x, t)}{|t|^{\theta}}>0$ uniformly in $x \in R^{N}$.

$\left(\mathrm{f}_{3}\right)$ There exist $\mu>2, L>0$, and $a \in\left[0, \frac{(\mu-2) V_{0}}{4}\right)$ such that

$$
\mu F(x, t)-f(x, t) t \leq a|t|^{2}, \quad \text { for a.e. } x \in R^{N} \text { and } \forall|t| \geq L .
$$

Now, we can state our result about the existence of a sequence of high energy solutions for the fourth-order elliptic equations (1.1) in $R^{N}$.

Theorem 1.1 Assume that $\left(\mathrm{V}_{1}\right),\left(\mathrm{f}_{1}\right)-\left(\mathrm{f}_{4}\right)$ hold. Then the problem (1.1) possesses a sequence of high energy solutions in $E$.

Remark 2 From Remark 1.1 in [26], we know that the condition $\left(f_{1}\right)$ is much weaker than the combination of the usual subcritical condition and the asymptotically linear condition near zero. Furthermore, conditions $\left(\mathrm{f}_{2}\right)-\left(\mathrm{f}_{3}\right)$ are much weaker than $\left(\mathrm{f}_{2}^{\prime}\right)$ - $\left(\mathrm{f}_{3}^{\prime}\right)$. Indeed, for any $x \in R^{N}, z \in R$, define

$$
h(t):=F\left(x, t^{-1} z\right) t^{\mu_{0}}, \quad \forall t \in[1,+\infty) .
$$

Then, for $|z| \geq L_{0}$ and $t \in\left[1, \frac{|z|}{L_{0}}\right]$, (f $\left.f_{3}^{\prime}\right)$ implies that

$$
h^{\prime}(t)=t^{\mu_{0}-1}\left[\mu_{0} F\left(x, t^{-1} z\right)-t^{-1} z f\left(x, t^{-1} z\right)\right] \leq 0 .
$$


Hence, $h(1) \geq h\left(\frac{|z|}{L_{0}}\right)$. Therefore, it follows from $\left(\mathrm{f}_{2}^{\prime}\right)$ that

$$
F(x, z) \geq F\left(x, \frac{L_{0} z}{|z|}\right)\left(\frac{|z|}{L_{0}}\right)^{\mu_{0}} \geq c|z|^{\mu_{0}}, \quad \forall x \in R^{N} \text { and }|z| \geq L_{0}
$$

where $c=\left(\frac{1}{L_{0}}\right)^{\mu_{0}} \inf _{x \in R^{N},|t|=L_{0}} F(x, t)>0$. Hence, (1.9) implies that $\left(\mathrm{f}_{2}\right)$ holds by taking $\theta=\mu_{0}$. Further, it follows from $\left(\mathrm{f}_{3}^{\prime}\right)$ that $\left(\mathrm{f}_{3}\right)$ holds by taking $L=L_{0}, \mu=\mu_{0}$. Consequently, $\left(f_{2}\right)-\left(f_{3}\right)$ are much weaker than $\left(f_{2}^{\prime}\right)-\left(f_{3}^{\prime}\right)$. Thus, Theorem 1.1 sharply improves Theorem A and, of course, unites and improves the results of [25].

\section{Some lemmas}

In order to apply variational techniques, we first state the key compactness result.

Lemma 2.1 (Lemma 3.4 in [31]) Under the assumption $\left(\mathrm{V}_{1}\right)$, the embedding

$$
E \hookrightarrow L^{s}\left(R^{N}\right), \quad 2 \leq s<2 *
$$

is compact.

The following lemma was proved by [26, Lemma 2.1].

Lemma 2.2 Assume that $\left(\mathrm{V}_{1}\right)$ and $\left(\mathrm{f}_{1}\right)$ hold. Then $I$ is well defined on $E, I \in C^{1}(E, R)$, and for any $u, v \in E$,

$$
\left\langle I^{\prime}(u), v\right\rangle=\int_{R^{N}}(\Delta u \Delta v+\nabla u \nabla v+V(x) u v) d x-\int_{R^{N}} f(x, u) v d x .
$$

Moreover, $\Psi^{\prime}: E \rightarrow E^{*}$ is compact, where $\Psi(u)=\int_{R^{N}} F(x, u) d x$.

Recall that we say $I$ satisfies the (PS) condition at the level $c \in R\left((\mathrm{PS})_{c}\right.$ condition for short) if any sequence $\left\{u_{n}\right\} \subset E$ along with $I\left(u_{n}\right) \rightarrow c$ and $I^{\prime}\left(u_{n}\right) \rightarrow 0$ as $n \rightarrow \infty$ possesses a convergent subsequence. If $I$ satisfies the $(\mathrm{PS})_{c}$ condition for each $c \in R$, then we say that I satisfies the (PS) condition.

Lemma 2.3 Let assumption $\left(\mathrm{V}_{1}\right)$ and $\left(\mathrm{f}_{1}\right)$ hold. Then any bounded Palais-Smale sequence of I has a strongly convergent subsequence in $E$.

Proof Let $\left\{u_{n}\right\} \subset E$ be any bounded Palais-Smale sequence of $I$. Then, up to a subsequence, there exists $c \in R$ such that

$$
I\left(u_{n}\right) \rightarrow c, \quad I^{\prime}\left(u_{n}\right) \rightarrow 0 \quad \text { and } \quad \sup _{n}\left\|u_{n}\right\|_{E}<+\infty
$$

Since the embedding

$$
E \hookrightarrow L^{s}\left(R^{N}\right), \quad 2 \leq s<2 *
$$


is compact, going if necessary to a subsequence, we can assume that there is a $u \in E$ such that

$$
\begin{cases}u_{n} \rightarrow u, & \text { weakly in } E ; \\ u_{n} \rightarrow u, & \text { strongly in } L^{s}\left(R^{N}\right) \\ u_{n}(x) \rightarrow u(x), & \text { a.e. in } R^{N} .\end{cases}
$$

In view of (2.1), one has

$$
\begin{aligned}
\left\langle I^{\prime}\left(u_{n}\right)-I^{\prime}(u), u_{n}-u\right\rangle= & \int_{R^{N}}\left(\Delta u_{n} \Delta\left(u_{n}-u\right)+\nabla u_{n} \nabla\left(u_{n}-u\right)+V(x) u_{n}\left(u_{n}-u\right)\right) d x \\
& -\int_{R^{N}} f\left(x, u_{n}\right)\left(u_{n}-u\right) d x \\
& -\int_{R^{N}}\left(\Delta u \Delta\left(u_{n}-u\right)-\nabla u \nabla\left(u_{n}-u\right)-V(x) u\left(u_{n}-u\right)\right) d x \\
& +\int_{R^{N}} f(x, u)\left(u_{n}-u\right) d x \\
= & \left\|u_{n}-u\right\|_{E}^{2}-\int_{R^{N}}\left[f\left(x, u_{n}\right)-f(x, u)\right]\left(u_{n}-u\right) d x .
\end{aligned}
$$

By $\left(f_{1}\right)$, using the Hölder inequality, we can conclude

$$
\begin{aligned}
\left|\int_{R^{N}}\left[f\left(x, u_{n}\right)-f(x, u)\right]\left(u_{n}-u\right) d x\right| \leq & C_{1} \int_{R^{N}}\left[\left|u_{n}\right|+|u|+\left|u_{n}\right|^{p-1}+|u|^{p-1}\right]\left|u_{n}-u\right| d x \\
\leq & C_{1}\left(\left\|u_{n}\right\|_{L^{2}}+\|u\|_{L^{2}}\right)\left\|u_{n}-u\right\|_{L^{2}} \\
& +C_{1}\left(\left\|u_{n}\right\|_{L^{p}}^{p-1}+\|u\|_{L^{p}}^{p-1}\right)\left\|u_{n}-u\right\|_{L^{p}} .
\end{aligned}
$$

Therefore, it follows from (2.3) that

$$
\int_{R^{N}}\left[f\left(x, u_{n}\right)-f(x, u)\right]\left(u_{n}-u\right) d x \rightarrow 0, \quad \text { as } n \rightarrow \infty .
$$

Moreover, combining (2.2) with (2.3), then

$$
\left\langle I^{\prime}\left(u_{n}\right)-I^{\prime}(u), u_{n}-u\right\rangle \rightarrow 0, \quad \text { as } n \rightarrow \infty
$$

Consequently, (2.4), (2.5) together with (2.6) imply that

$$
u_{n} \rightarrow u, \quad \text { strongly in } E \text { as } n \rightarrow \infty .
$$

This completes the proof.

Lemma 2.4 Let assumptions $\left(\mathrm{V}_{1}\right),\left(\mathrm{f}_{1}\right)$, and $\left(\mathrm{f}_{3}\right)$ hold. Then any Palais-Smale sequence of I is bounded.

Proof Let $\left\{u_{n}\right\} \subset E$ be any Palais-Smale sequence of $I$, then, up to a subsequence, there exists $c_{1} \in R$ such that

$$
I\left(u_{n}\right) \rightarrow c_{1}, \quad \text { and } \quad I^{\prime}\left(u_{n}\right) \rightarrow 0 .
$$


The combination of (1.7), (1.8), (2.1), (2.7), $\left(\mathrm{V}_{1}\right)$ with $\left(\mathrm{f}_{3}\right)$ implies

$$
\begin{aligned}
c_{1}+ & +\left\|u_{n}\right\|_{E} \\
\geq & I\left(u_{n}\right)-\frac{1}{\mu}\left\langle I^{\prime}\left(u_{n}\right), u_{n}\right\rangle \\
= & \frac{\mu-2}{2 \mu} \int_{R^{N}}\left(\left|\Delta u_{n}\right|^{2}+\left|\nabla u_{n}\right|^{2} d x+\int_{R^{N}} V(x) u_{n}^{2}\right) d x+\int_{R^{N}} \widetilde{F}\left(x, u_{n}\right) d x \\
\geq & \frac{\mu-2}{2 \mu} \int_{R^{N}}\left(\left|\Delta u_{n}\right|^{2}+\left|\nabla u_{n}\right|^{2} d x+\int_{R^{N}} V(x) u_{n}^{2}\right) d x \\
& -\frac{a}{\mu} \int_{R^{N}} u_{n}^{2} d x+\int_{A_{n}} \widetilde{F}\left(x, u_{n}\right) d x \\
\geq & \frac{\mu-2}{2 \mu} \int_{R^{N}}\left(\left|\Delta u_{n}\right|^{2}+\left|\nabla u_{n}\right|^{2} d x+\int_{R^{N}} V(x) u_{n}^{2}\right) d x \\
& -\frac{a}{\mu V_{0}} \int_{R^{N}} V(x) u_{n}^{2} d x+\int_{A_{n}} \widetilde{F}\left(x, u_{n}\right) d x \\
\geq & \frac{\mu-2}{4 \mu}\left\|u_{n}\right\|_{E}^{2}+\left(\frac{\mu-2}{4 \mu}-\frac{a}{\mu V_{0}}\right) \int_{R^{N}} V(x) u_{n}^{2} d x+\int_{A_{n}} \widetilde{F}\left(x, u_{n}\right) d x \\
= & \frac{\mu-2}{4 \mu}\left\|u_{n}\right\|_{E}^{2}+\frac{(\mu-2) V_{0}-4 a}{4 \mu V_{0}} \int_{R^{N}} V(x) u_{n}^{2} d x+\int_{A_{n}} \widetilde{F}\left(x, u_{n}\right) d x,
\end{aligned}
$$

where $\widetilde{F}\left(x, u_{n}\right)=\frac{1}{\mu} f\left(x, u_{n}\right) u_{n}-F\left(x, u_{n}\right)$ and $A_{n}=\left\{x \in R^{N}:\left|u_{n}\right| \leq L\right\}$. For $x \in R^{N}$ and $\left|u_{n}\right| \leq L$, by $\left(\mathrm{f}_{1}\right)$, one has

$$
\begin{aligned}
\left|\widetilde{F}\left(x, u_{n}\right)\right| & \leq \frac{1}{\mu}\left|f\left(x, u_{n}\right)\right|\left|u_{n}\right|+\left|F\left(x, u_{n}\right)\right| \\
& \leq \frac{1}{\mu} C_{1}\left(\left|u_{n}\right|^{2}+\left|u_{n}\right|^{p}\right)+C_{1}\left(\frac{1}{2}\left|u_{n}\right|^{2}+\frac{1}{p}\left|u_{n}\right|^{p}\right) \\
& \leq C_{1}\left(1+\left|u_{n}\right|^{p-2}\right)\left|u_{n}\right|^{2} \\
& \leq C_{1}\left(1+L^{p-2}\right)\left|u_{n}\right|^{2} .
\end{aligned}
$$

Take $M>\max \left\{\frac{4 C_{1}\left(1+L^{p-2}\right) \mu V_{0}}{(\mu-2) V_{0}-4 a}, V_{0}\right\}$, then

$$
\widetilde{F}\left(x, u_{n}\right) \geq-\frac{(\mu-2) V_{0}-4 a}{4 \mu V_{0}} M\left|u_{n}\right|^{2}, \quad \forall x \in R^{N},\left|u_{n}\right| \leq L .
$$

Let $\widetilde{A}=\left\{x \in R^{N}: V(x) \leq M\right\}, R(n)=\frac{(\mu-2) V_{0}-4 a}{4 \mu V_{0}} \int_{R^{N}} V(x) u_{n}^{2} d x+\int_{A_{n}} \widetilde{F}\left(x, u_{n}\right) d x$. By $\left(\mathrm{V}_{1}\right)$ and (2.9), we can conclude

$$
\begin{aligned}
R(n) & \geq \frac{(\mu-2) V_{0}-4 a}{4 \mu V_{0}} \int_{\left|u_{n}\right| \leq L}(V(x)-M)\left|u_{n}\right|^{2} d x \\
& \geq \frac{(\mu-2) V_{0}-4 a}{4 \mu V_{0}} \int_{\widetilde{A} \cap A_{n}}(V(x)-M) L^{2} d x \\
& \geq \frac{(\mu-2) V_{0}-4 a}{4 \mu V_{0}}\left(V_{0}-M\right) L^{2} \operatorname{meas}\left(\tilde{A} \cap A_{n}\right) \\
& \geq \frac{(\mu-2) V_{0}-4 a}{4 \mu V_{0}}\left(V_{0}-M\right) L^{2} \operatorname{meas}(\tilde{A}) .
\end{aligned}
$$


Note that meas $(\tilde{A})<+\infty$ due to $\left(\mathrm{V}_{1}\right)$, and it follows from $(2.8)$ and $(2.10)$ that

$$
\begin{aligned}
c_{1}+1+\left\|u_{n}\right\|_{E} & \geq \frac{\mu-2}{4 \mu}\left\|u_{n}\right\|_{E}^{2}+R(n) \\
& \geq \frac{\mu-2}{4 \mu}\left\|u_{n}\right\|_{E}^{2}+\frac{(\mu-2) V_{0}-4 a}{4 \mu V_{0}}\left(V_{0}-M\right) L^{2} \operatorname{meas}(\widetilde{A}),
\end{aligned}
$$

which implies $\left\{u_{n}\right\} \subset E$ is bounded in $E$. Hence the proof is completed.

Remark 3 We present a new proof technique to verify the boundedness of Palais-Smale sequences, which is much clearer and simpler than the previous literature.

\section{Proof of Theorem 1.1}

In this section we will use the classical symmetric mountain pass theorem of Rabinowitz instead of the fountain theorem in [26] to obtain high energy solutions for the problem (1.1) and prove Theorem 1.1. First of all, we present some notations.

In view of $E \hookrightarrow L^{2}\left(R^{N}\right)$ and $L^{2}\left(R^{N}\right)$ being a separable Hilbert space, $E$ has a countable orthogonal basis $\left\{e_{j}\right\}_{j=1}^{\infty}$. Let

$$
E_{k}=\bigoplus_{j=1}^{k} X_{j}, \quad Z_{k}=E_{k}^{\perp}
$$

where $X_{j}=\operatorname{span}\left\{e_{j}\right\}$. Thus, $E=E_{k} \oplus Z_{k}$ and $E_{k}$ is finite dimensional.

Lemma 3.1 Let the assumption $\left(\mathrm{V}_{1}\right)$ hold. Define

$$
\eta(k):=\sup _{u \in Z_{k},\|u\| \|_{E}=1}\|u\|_{L^{2}}, \quad k \in N
$$

then there exists $k_{0} \in N$ such that $0<\eta\left(k_{0}\right) \leq\left(\frac{1}{2 C_{1}}\right)^{\frac{1}{2}}$.

Proof Firstly, $\eta(k)$ is convergent since $\eta(k) \geq 0$ and $\eta(k)$ is decreasing in $k$. Furthermore, for any $k \in N$, by the definition of $\eta(k)$, there exists $u_{k} \in Z_{k}$ such that

$$
\left\|u_{k}\right\|_{E}=1 \quad \text { and } \quad\left\|u_{k}\right\|_{L^{2}}>\frac{\eta(k)}{2} .
$$

For any $v \in E, v=\sum_{n=1}^{\infty} a_{n} e_{n}$, it has

$$
\begin{aligned}
\left|\left\langle u_{k}, v\right\rangle_{E}\right| & =\left|\left\langle u_{k}, \sum_{n=1}^{\infty} a_{n} e_{n}\right\rangle_{E}\right| \leq\left\|u_{k}\right\|_{E}\left\|\sum_{n=k+1}^{\infty} a_{n} e_{n}\right\|_{E} \\
& \leq\left\|\sum_{n=k+1}^{\infty} a_{n} e_{n}\right\|_{E} \rightarrow 0
\end{aligned}
$$

as $k \rightarrow \infty$, which implies that $u_{k} \rightarrow 0$ weakly in $E$. By virtue of Lemma 2.1, we can conclude

$$
u_{k} \rightarrow 0 \quad \text { strongly in } L^{2}\left(R^{N}\right)
$$


The combination (3.1) with (3.2) implies that $\eta(k) \rightarrow 0$, as $k \rightarrow \infty$. Then there exists $k_{0} \in$ $N$ such that $0<\eta\left(k_{0}\right) \leq\left(\frac{1}{2 C_{1}}\right)^{\frac{1}{2}}$. Hence the proof is completed.

Lemma 3.2 Let assumptions $\left(\mathrm{V}_{1}\right)$ and $\left(\mathrm{f}_{1}\right)$ hold, then there exist some constants $\rho$, $\alpha$ such that $I(u) \geq \alpha$ whenever $u \in Z_{k_{0}}$ with $\|u\|_{E}=\rho$.

Proof For any $u \in Z_{k_{0}}$, by Lemma 3.1, we have

$$
\|u\|_{L^{2}} \leq \eta\left(k_{0}\right)\|u\|_{E} \quad \text { and } \quad 0<\eta\left(k_{0}\right) \leq\left(\frac{1}{2 C_{1}}\right)^{\frac{1}{2}}
$$

By (1.7), (1.8), ( $\left.f_{1}\right),(3.3)$, and the Hölder inequality, one has

$$
\begin{aligned}
I(u) & =\frac{1}{2}\|u\|_{E}^{2}-\int_{R^{N}} F(x, u) d x \\
& \geq \frac{1}{2}\|u\|_{E}^{2}-\frac{C_{1}}{2}\left(\|u\|_{L^{2}}^{2}+\|u\|_{L^{p}}^{p}\right) \\
& \geq \frac{1}{2}\|u\|_{E}^{2}-\frac{C_{1}}{2} \eta^{2}\left(k_{0}\right)\|u\|_{E}^{2}-\frac{C_{1}}{2} a_{p}^{p}\|u\|_{E}^{p} \\
& \geq \frac{1}{2}\|u\|_{E}\left[\frac{1}{2}\|u\|_{E}-C_{1} a_{p}^{p}\|u\|_{E}^{p-1}\right] .
\end{aligned}
$$

Set

$$
l(t)=\frac{1}{2} t-C_{1} a_{p}^{p} t^{p-1}, \quad \forall t \geq 0 .
$$

Note that $p>2$, we can conclude that there exists a constant $\rho>0$ such that

$$
l(\rho)=\max _{t \geq 0} l(t)>0 .
$$

Therefore,

$$
I(u) \geq \frac{1}{2} \rho l(\rho)=: \alpha>0,
$$

whenever $u \in Z_{k_{0}}$ with $\|u\|_{E}=\rho$. This completes the proof.

Lemma 3.3 Let assumptions $\left(\mathrm{f}_{1}\right)-\left(\mathrm{f}_{2}\right)$ hold, then for each finite dimensional subspace $\widetilde{E} \subset E$, there is an $r=r(\widetilde{E})>0$ such that $\left.I\right|_{\widetilde{E} \backslash B_{r}}<0$.

Proof By the assumptions $\left(\mathrm{f}_{1}\right)-\left(\mathrm{f}_{2}\right)$, there exist two positive constants $C_{2}$ and $C_{3}$ such that

$$
F(x, z) \geq C_{2}|z|^{\theta}-C_{3}|z|^{2}, \quad \forall(x, z) \in R^{N} \times R .
$$

For any finite dimensional subspace $\widetilde{E} \subset E$, by the equivalence of norms in the finite dimensional space, there exists a constant $\beta_{s}>0$ such that

$$
\|u\|_{L^{s}} \geq \beta_{s}\|u\|_{E}, \quad \forall u \in \widetilde{E}
$$


for $2 \leq s<2_{*}$. Therefore, the combination of (1.7)-(1.8) with (3.4)-(3.5) implies

$$
\begin{aligned}
I(u) & =\frac{1}{2}\|u\|_{E}^{2}-\int_{R^{N}} F(x, u) d x \\
& \leq \frac{1}{2}\|u\|_{E}^{2}-C_{2}\|u\|_{L^{\theta}}^{\theta}+C_{3}\|u\|_{L^{2}}^{2} \\
& \leq \frac{1}{2}\|u\|_{E}^{2}-C_{2} \beta_{\theta}^{\theta}\|u\|_{E}^{\theta}+C_{3} a_{2}^{2}\|u\|_{E}^{2}
\end{aligned}
$$

for all $u \in \widetilde{E}$. Note that $\theta>2$, hence there is an $r=r(\widetilde{E})>0$ such that $\left.I\right|_{\widetilde{E} \backslash B_{r}}<0$. This completes the proof.

Next, we shall prove our Theorem 1.1. To begin with, for convenience of notation, we state the classical symmetric mountain pass theorem as follows.

Theorem 3.4 ([32, Theorem 9.12]) Let E be an infinite dimensional Banach space, and let $I \in C^{1}(E, R)$ be even and satisfy the $(P S)$ condition and $I(0)=0$. If $E=Y \oplus Z, Y$ is finite dimensional, and I satisfies

( $\left.\mathrm{I}_{1}\right)$ there exist constants $\rho, \alpha>0$ such that $\left.I\right|_{\partial B_{\rho} \cap Z} \geq \alpha$, and

$\left(\mathrm{I}_{2}\right)$ for each finite dimensional subspace $\widetilde{E} \subset E$, there is $r=r(\widetilde{E})$ such that $I \leq 0$ on $\widetilde{E} \backslash B_{r}$,

then I possesses an unbounded sequence of critical values.

Proof of Theorem 1.1 The proof is to verify $I$ satisfies all the conditions of Theorem 3.4. Set $Y=E_{k_{0}}, Z=Z_{k_{0}}$, then $E=Y \oplus Z$ and $Y$ is finite dimensional. First, $I$ satisfies $\left(\mathrm{I}_{1}\right)$ and $\left(\mathrm{I}_{2}\right)$ in Theorem 3.4 by Lemmas 3.2 and 3.3, respectively. Second, $I$ satisfies the (PS) condition by virtue of Lemmas 2.3 and 2.4. Finally, $I(0)=0, I$ is even on $E$ due to $\left(\mathrm{f}_{4}\right)$ and $I \in C^{1}(E, R)$ by Lemma 2.2. Hence, the conclusion directly follows from Theorem 3.4. The proof is completed.

Remark 4 Compared with Theorem A (see [26, Theorem 1.1]), on one hand, the assumptions imposed on $f$ are much weaker. On the other hand, we present a new proof technique to verify the boundedness of Palais-Smale sequences, and we apply the classical symmetric mountain pass theorem of Rabinowitz instead of the fountain theorem in [26] to obtain high energy solutions for the problem (1.1). Hence, it is very different.

Competing interests

The author declares that he has no competing interests.

\section{Acknowledgements}

This work is partly supported the Foundation of Education of Commission of Yunnan Province (2014Z153, 2012Z123C), and the Youth Program of Yunnan Provincial Science and Technology Department (2013FD046).

Received: 15 June 2014 Accepted: 7 August 2014 Published online: 25 September 2014

\section{References}

1. Lazer, AC, Mckenna, PJ: Large-amplitude periodic oscillations in suspension bridges: some new connections with nonlinear analysis. SIAM Rev. 32(4), 537-578 (1990)

2. Lazer, AC, Mckenna, PJ: Global bifurcation and a theorem of Tarantello. J. Math. Anal. Appl. 181(3), 648-655 (1994)

3. Tarantella, G: A note on a semilinear elliptic problem. Differ. Integral Equ. 5(3), 561-565 (1992)

4. Mckenna, PJ, Walter, W: Traveling waves in a suspension bridge. SIAM J. Appl. Math. 50, 703-715 (1990) 
5. Chen, Y, Mckenna, PJ: Traveling waves in a nonlinear suspension beam: theoretical results and numerical observations. J. Differ. Equ. 135, 325-355 (1997)

6. Pao, CV: On fourth-order elliptic boundary value problems. Proc. Am. Math. Soc. 128, 1023-1030 (2000)

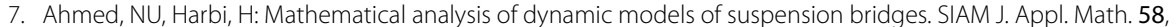
853-874 (1998)

8. Micheletti, AM, Pistoia, A: Multiplicity results for a fourth-order semilinear elliptic problem. Nonlinear Anal. 31(7), 895-908 (1998)

9. Micheletti, AM, Pistoia, A: Nontrivial solutions for some fourth order semilinear elliptic problems. Nonlinear Anal. 34(4), 509-523 (1998)

10. Zhang, J: Existence results for some fourth-order nonlinear elliptic problems. Nonlinear Anal. 45(1), $29-36$ (2001)

11. $\mathrm{Xu}, \mathrm{G}$, Zhang, J: Existence results for some fourth-order nonlinear elliptic problems of local superlinearity and sublinearity. J. Math. Anal. Appl. 281(2), 633-640 (2003)

12. Zhang, J, Li, S: Multiple nontrivial solutions for some fourth-order semilinear elliptic problems. Nonlinear Anal. 60(2), 221-230 (2005)

13. Wang, W, Zang, A, Zhao, P: Multiplicity of solutions for a class of fourth-order elliptic equations. Nonlinear Anal. 70(12), 4377-4385 (2009)

14. Zhou, J, Wu, X: Sign-changing solutions for some fourth-order nonlinear elliptic problems. J. Math. Anal. Appl. 342(1), 542-558 (2008)

15. Wang, Y, Shen, Y: Infinitely many sign-changing solutions for a class of biharmonica equation without symmetry. Nonlinear Anal. 71, 967-977 (2009)

16. Yang, Y, Zhang, J: Existence of solutions for some fourth-order nonlinear elliptic problems. J. Math. Anal. Appl. 351(1), 128-137 (2009)

17. Liu, $X$, Huang, Y: On sign-changing solutions for a fourth-order asymptotically linear elliptic problems. Nonlinear Anal. 72(5), 2271-2276 (2010)

18. Qian, AX, Li, SJ: Multiple solutions for a fourth-order asymptotically linear elliptic problems. Acta Math. Sin. Engl. Ser. 22(4), 1121-1126 (2006)

19. An, Y, Liu, R: Existence of nontrivial solutions of an asymptotically linear fourth-order elliptic equations. Nonlinear Anal. 68(11), 3325-3331 (2008)

20. Zhang, J, Wei, Z: Infinitely many nontrivial solutions for a class of biharmonic equations via variant fountain theorems Nonlinear Anal. 74, 7474-7485 (2011)

21. Zhang, J, Wei, Z: Multiple solutions for a class of biharmonic equations with a nonlinearity concave at the origin J. Math. Anal. Appl. 383, 291-306 (2011)

22. Lü, D: Multiple solutions for a class of biharmonic elliptic system with Sobolev critical exponent. Nonlinear Anal. 74(17), 6371-6382 (2011)

23. Chabrowski, J, Marcos do Ó, J: On some fourth-order semilinear elliptic problems in $R^{N}$. Nonlinear Anal. 49, 861-884 (2002)

24. Pimenta, MTO, Soares, SHM: Existence and concentration of solutions for a class biharmonic equation. J. Math. Anal. Appl. 390, 274-289 (2012)

25. Yin, $Y, W u, X$ : High energy solutions and nontrivial solutions for fourth-order elliptic equations. J. Math. Anal. Appl. $375,699-705(2011)$

26. Ye, Y, Tang, C: Infinitely many solutions for fourth-order elliptic equations. J. Math. Anal. Appl. 394, 841-854 (2012)

27. Lin, J, Chen, S, Wu, X: Existence and multiplicity of solutions for a class of fourth-order elliptic equations in $R^{N}$. J. Math. Anal. Appl. 395, 608-615 (2012)

28. Ye, $Y$, Tang, C: Existence and multiplicity of solutions for fourth-order elliptic equations in $R^{N}$. J. Math. Anal. Appl. 406 335-351 (2013)

29. Zhang, W, Tang, X, Zhang, J: Infinitely many solutions for fourth-order elliptic equations with general potentials. J. Math. Anal. Appl. 407, 359-368 (2013)

30. Bartsch, T, Wang, ZQ: Existence and multiplicity results for some superlinear elliptic problems on $R^{N}$. Commun. Partial Differ. Equ. 20, 1725-1741 (1995)

31. Zou, WM, Schechter, M: Critical Point Theory and Its Applications. Springer, New York (2006)

32. Rabinowitz, PH: Minimax Methods in Critical Point Theory with Application to Differential Equations. CBMS Regional Conf. Ser. in Math., vol. 65. Am. Math. Soc., Providence (1986)

doi:10.1186/s13661-014-0199-y

Cite this article as: Cheng: High energy solutions for the fourth-order elliptic equations in $R^{N}$. Boundary Value Problems 2014 2014:199. 